\title{
GAIA Low Birth Weight Level of Diagnostic Certainty
}

National Cancer Institute

\section{Source}

National Cancer Institute. GAIA Low Birth Weight Level of Diagnostic Certainty. NCI

Thesaurus. Code C128675.

A classification of maternal and fetal outcomes relating to low birth weight, developed by the Global Alignment of Immunization safety Assessment in pregnancy, based on the extent to which the diagnosis has been confirmed. 